\title{
Laryngeal Nerve Paralysis
}

National Cancer Institute

\section{Source}

National Cancer Institute. Laryngeal Nerve Paralysis. NCI Thesaurus. Code C78591.

Damage to the laryngeal nerve that results in paralysis of the layrnx. 